\title{
Systematic review of neurotrophic tropomyosin-related kinase inhibition as a tumor-agnostic management strategy
}

\author{
Paula Chu*,1, Sarah Batson², Matthew Hodgson³, Catherine R Mitchell ${ }^{2}$ (iD \& Anna \\ Steenrod ${ }^{1}$ \\ ${ }^{1}$ F Hoffmann-La Roche Ltd., Global Access, 4070 Basel, Switzerland \\ ${ }^{2}$ Mtech Access Ltd., 30 Murdock Road, Bicester, OX26 4PP, UK \\ ${ }^{3}$ Roche Products Ltd., Health Economics and Strategic Pricing, Welwyn Garden City, AL7 1TW, UK \\ *Author for correspondence: paula.chu@roche.com
}

\begin{abstract}
Aim: To conduct a systematic review and meta-analysis feasibility of clinical, quality of life and economic evidence for neurotrophic tropomyosin-related receptor tyrosine kinases (NTRK) inhibitors in patients with NTRK gene fusion-positive tumors. Materials \& methods: Databases were searched for studies on NTRK inhibitors in adult and pediatric patients. Results: 27 publications reported clinical data for seven interventions. Efficacy/safety data were available for two interventions only. Four trials each reported data for larotrectinib and entrectinib with pooled analyses reporting objective response rates of $75 \%(95 \% \mathrm{Cl}$ : 61-85) and $57.4 \%$ (43.2-70.8), respectively. No publications reported economic or quality of life evidence. Conclusion: Preliminary data demonstrate that NTRK inhibitors are well tolerated and show impressive clinical benefit; corroboration of existing studies and real-world data are required.
\end{abstract}

First draft submitted: 2 September 2019; Accepted for publication: 11 December 2019; Published online: 16 January 2020

Keywords: NTRK • cabozantinib • entrectinib • larotrectinib • merestinib • neurotrophic tyrosine kinase receptors $\bullet$ systematic review $\bullet$ TKI $\bullet$ tyrosine kinase inhibitor

Tumor-agnostic therapy, whereby treatment is tailored to match specific gene alterations regardless of tumor location is becoming increasingly common [1]. The introduction of biomarkers into drug development has led to multiple new challenges stretching from the implementation of biomarker screening approaches to the development of appropriate market access reimbursement strategies. One such set of biomarkers are gene fusions involving the neurotrophic tropomyosin-related kinases (NTRK).

Fusions in the NTRK1, NTRK2 and NTRK3 genes, which are thought to be present in up to $1 \%$ of all solid tumors, are found in a wide range of adult and pediatric tumor types [2,3]. The genes encode neurotrophic tyrosine kinase receptors (TRK, or tropomyosin-related receptor tyrosine kinases) TRKA, TRKB and TRKC, which in a normal state contribute to neuronal development, function, survival and proliferation during childhood and into adulthood. However, in a rearranged state, fusions may activate signal transduction leading to oncogenesis [4]. TRK fusions resulting from $N T R K 1 / 2 / 3$ genetic alterations drive oncogenic transformations in more than 20 different malignancies over diverse tissue/cell lineages, in both the pediatric and adult populations [5]. These NTRK fusions provide novel therapeutic anticancer targets and several Phase I and II trials of 'tumor-agnostic' tropomyosin-related receptor tyrosine kinase inhibitors (TKI) are ongoing to assess their potential clinical effectiveness in patients with tumors harboring NTRK gene fusions [6]. Several TKIs with varying degrees of activity against TRKA, TRKB and/or TRKC are available, which can broadly be grouped into multikinase inhibitors with activity against a range of targets including TRK or more selective TRK inhibitors [7]. The multikinase inhibitor group includes entrectinib, repotrectinib (formally known as ropotrectinib), cabozantinib, merestinib, MGCD516, PLX7486, DS-6051b and TSR-011 [2]. Larotrectinib and entrectinib both selective TRK inhibitors have recently been granted accelerated approval from the US FDA as tumor-agnostic therapies [8,9]. Due to the rarity of NTRK fusions in single tumor types, nonrandomized controlled trial (RCT) study designs such as basket trials, whereby the effect of one drug on

Future Medicine 
a single mutation in a variety of tumor types is considered, are more commonplace. This leads to challenges when seeking reimbursement where RCT study designs are favored by health technology assessment (HTA) bodies.

In order to identify patients who would benefit from TKI therapy, several methods of detecting TRK fusions in cancer are currently in use. Initially, most fusions were detected by fluorescence in situ hybridization probes, which is a rapid and relatively inexpensive method to identify TRK fusions and appears to be highly specific in tumor histologies with a high incidence of TRK fusion. More recently, clinical grade next-generation sequencing has been employed to detect TRK fusions [10].

The aim of the current systematic review is to provide a summary of published evidence for the currently available TRK-targeted agents. End points considered for inclusion were clinical, economic, quality of life (QoL) and cost/resource use outcomes. On completion of the clinical systematic review, the feasibility of conducting meta-analyses to allow a quantitative comparison of TRK-targeted agents for outcomes of interest was conducted.

\section{Materials \& methods}

Systematic literature searches were performed in October 2018 (to identify clinical, economic evaluation and QoL publications) and in January 2019 (to identify cost/resource use publications) in adherence with the Preferred Reporting Items for Systematic Reviews and Meta-Analyses statement [11]. Subject headings and keywords were used to identify English language publications reporting on the use of TRK-targeted agents with no restriction on solid tumor type in the following electronic databases: Medline ${ }^{\circledR}$, Medline ${ }^{\circledR}$ Epub Ahead of Print (In-Process \& Other Non-Indexed Citations), Embase and EBM Reviews. Additional searches of congress proceedings from the past 3 years, reference lists of included publications, relevant HTA bodies and clinical trial registries were conducted to identify relevant evidence. Eligibility criteria included clinical studies of any design (with the exception of single-patient case reports), economic evaluations, QoL studies or cost/resource use studies conducted in patients (adults or pediatric patients) with NTRK-fusion-positive solid tumors. Interventions of interest (clinical and economic evaluation systematic reviews only) were restricted to the following TKIs currently studied in this indication: entrectinib, larotrectinib, ropotrectinib, cabozantinib, LOXO-195, ONO-7579, sitravatinib and merestinib. Records were reviewed based on title and abstract in the first instance, and those included were reviewed based on the full publication. Records were reviewed by an analyst and independently checked by a second analyst, with any discrepancies resolved by consensus. This procedure complies with HTA guidelines for conducting a robust systematic review [12]. Quality (risk of bias) assessment of primary studies published as a full article was conducted using a quality appraisal checklist, as recommended by the National Institute for Health and Care Excellence (NICE) for the assessment of quantitative intervention studies [13].

Full details of the eligibility criteria and the electronic database search strategies search strategies are provided as Supplementary data.

A meta-analysis feasibility assessment was conducted using data from the identified publications to ascertain whether it was possible to generate indirect estimates of relative treatment effects across the TRK-targeted agents in all-comers, stratified by tumor type. Outcomes of interest included overall survival (OS), progression-free survival (PFS) and objective response rate (ORR). The feasibility included an assessment of heterogeneity across the studies included in the outcome-specific networks in terms of differences in study design and enrolled patient characteristics.

\section{Results}

Study inclusion \& characteristics

The electronic database search to identify clinical, economic evaluation and QoL publications (October 2018) identified a total of 3244 articles. After the removal of 599 duplicates, 2645 articles were screened by title and abstract. In total, 2565 articles were excluded, and 80 articles were deemed potentially relevant. After the review of the full publications, a further 39 articles were excluded and 19 single-patient case reports were tagged. Handsearching identified a further five relevant clinical studies and therefore a total of 27 relevant clinical studies were eligible for inclusion in the systematic review [14-40]. No relevant economic evaluations or QoL studies were identified (Figure 1). The electronic database search to identify cost and resource use publications (January 2019) retrieved a total of 183 articles for screening on the basis of title and abstract (following removal of a single duplicate). However, no relevant cost/resource use studies (in any territory) were identified (Figure 2). 


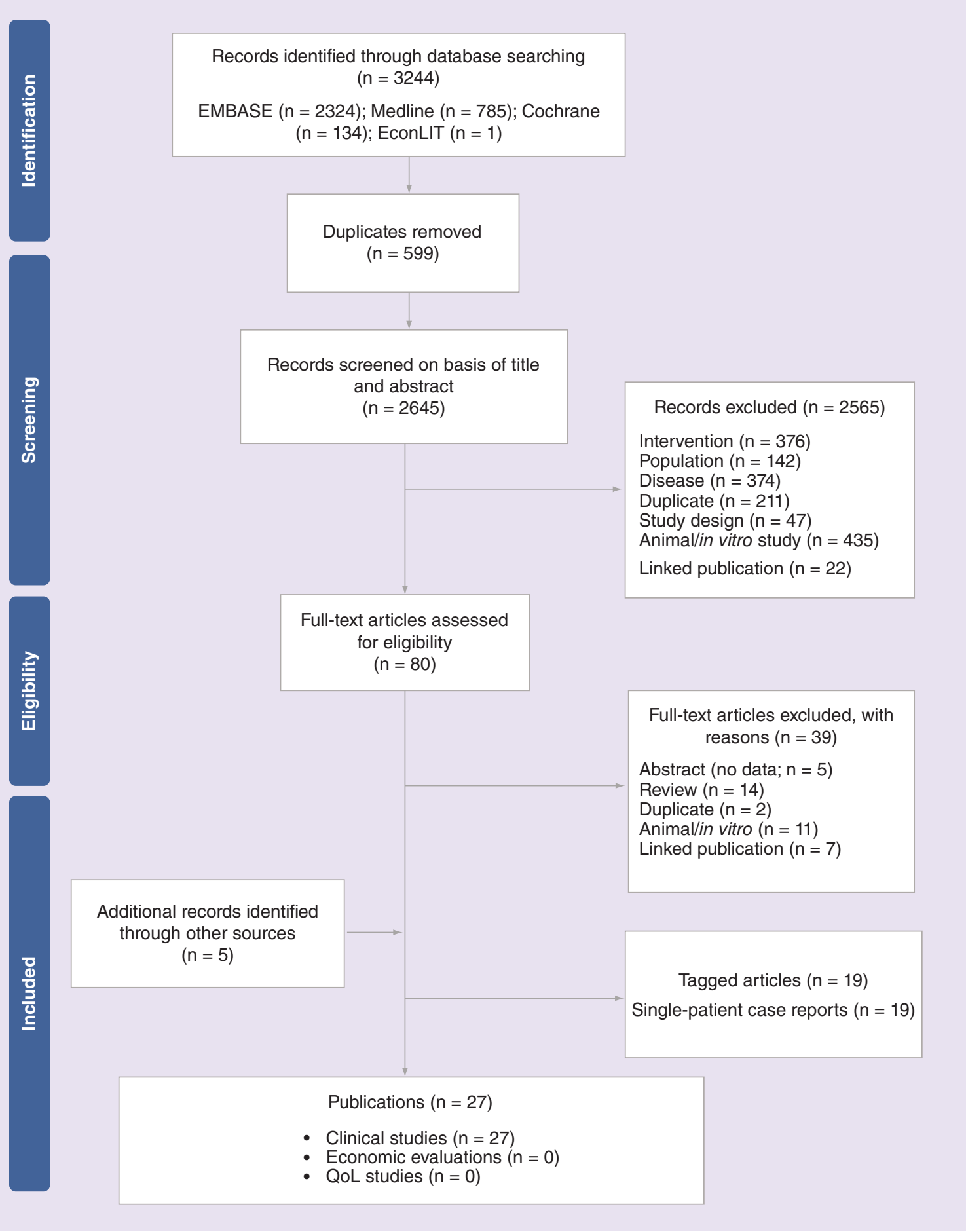

Figure 1. Preferred reporting items for systematic reviews and meta-analyses flow diagram for clinical, economic evaluation, quality of life systematic review.

QoL: Quality of life.

\section{Clinical studies}

The 27 publications reporting on clinical studies that met the inclusion criteria for the systematic review were associated with seven relevant interventions [14-40]. All clinical studies were of a single-arm trial design. The largest published evidence base was available for larotrectinib where 14 publications (reporting on four unique 

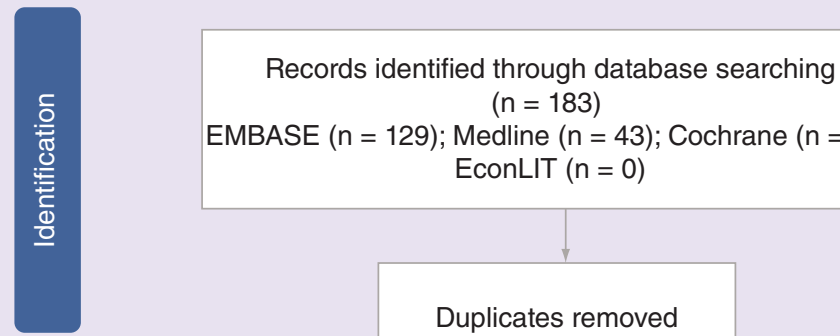

$$
(n=183)
$$

EMBASE $(n=129)$; Medline $(n=43)$; Cochrane $(n=11)$;

EconLIT $(n=0)$

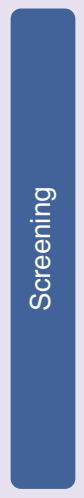

Duplicates removed

$$
(n=1)
$$

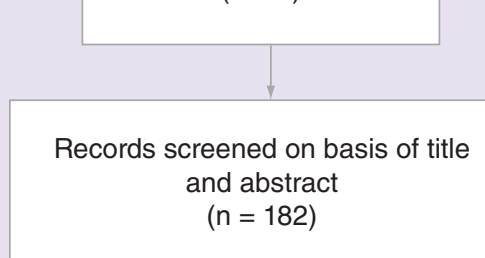

Records excluded $(n=182)$

Population $(\mathrm{n}=8)$

Duplicate $(n=54)$

Study design $(n=12)$

Animal/in vitro study $(\mathrm{n}=66)$

Review/editorial $(n=42)$

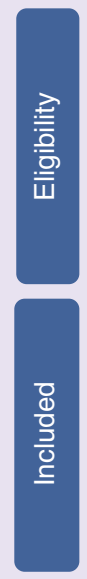

Full-text articles assessed for eligibility $(\mathrm{n}=0)$

Publications $(\mathrm{n}=0)$

Figure 2. Preferred reporting items for systematic reviews and meta-analyses flow diagram for cost/resource use systematic review.

studies) were identified $[15,20,21,27-34,37,38,40]$, followed by entrectinib with seven publications (of four unique trials) $[16-19,25,26,35]$. There was a general paucity of data for other targeted agents with only two publications reporting on belizatinib and one each for LOXO-195 [22], cabozantinib [23], repotrectinib [24] and sitravatinib (MGCD516) [14]. Clinical efficacy/safety data were only available for three interventions: larotrectinib [15,20,21,2734,37,38,40], entrectinib [16-19,25,26,35] and ropotrectinib (Table 1 [24] \& Supplementary information) with only early-stage pharmacokinetic and dose-finding data being reported for the other interventions [14,22,23,24].

\section{Larotrectinib}

Four trials reported data for larotrectinib in patients with NTRK mutations: NCT02122913 (Phase I) $[15,21,29,30,32,34,40,41]$, SCOUT (NCT02637687; Phase I/II) [21,27,28,30-34,38,40], NAVIGATE (NCT02576431; Phase II) $[20,21,30,32,34,40]$, NCT03025360 (expanded access program) [37]. At the time of the review, a fifth trial (MATCH, NCT03213704; Phase II) was recruiting pediatric patients and therefore no results were available. Of the five trials, two exclusively enrolled patients with $N T R K$-fusion-positive solid tumors (NAVIGATE and NCT03025360) and the other three recruited patients with a range of mutations (i.e., not limited to NTRK- 
Table 1. Published pooled analyses of clinical trials investigating the efficacy and safety of TRK-targeted agents in patients with NTRK gene fusions.

\begin{tabular}{|c|c|c|c|c|}
\hline Study, study design & Patient population & $\begin{array}{l}\text { No. of participants (no. } \\
\text { with NTRK fusion) }\end{array}$ & Outcome data reported for NTRK fusion population & Ref. \\
\hline \multicolumn{5}{|l|}{ Larotrectinib } \\
\hline $\begin{array}{l}\text { Pooled analysis } \\
\text { (NCT02122913, } \\
\text { SCOUT and } \\
\text { NAVIGATE) }\end{array}$ & $\begin{array}{l}\text { NTRK fusion patients with RECIST } \\
\text { measurable disease enrolled to the } \\
\text { adult (NCT02122913, } \mathrm{n}=8 \text { ) and } \\
\text { pediatric (SCOUT, NCT02637687, } \\
\mathrm{n}=12 \text { ) Phase I trials and } \\
\text { adult/adolescent Phase II trial } \\
\text { (NAVIGATE, NCT02576431, } \mathrm{n}=35 \text { ) }\end{array}$ & $55(55)$ & $\begin{array}{l}\text { - ORR, } 75 \%(95 \% \mathrm{Cl}: 61-85)^{\dagger} \\
\text { - ORR, } 80 \%(95 \% \mathrm{Cl}: 67-90)^{\ddagger} \\
\text { After } 1 \text { year: } \\
\text { - } 71 \% \text { were still alive } \\
\text { Safety } \\
\text { - Most AEs were Grade } 1 / 2 \text { and reversible } \\
\text { - Most common Grade } 3, \mathrm{Grade} 4, \text { any treatment-related AEs: } \\
\text { - Increased ALT or AST level, } 5,0,38 \% \\
\text { - Dizziness, } 2,0,25 \% \\
\text { - Nausea, } 2,0,16 \% \\
\text { - Anemia, } 2,0,9 \% \\
\text { - Decreased neutrophil count, } 2,0,9 \% \\
\text { - Fatigue, } 0,0,16 \% \\
\text { - Constipation, } 0,0,16 \% \\
\text { - Weight gain, } 0,0,11 \% \\
\text { - Treatment-related AEs leading to } \\
\text { - Dose reduction, } 15 \% \\
\text { - Discontinuation in patients with response to treatment, } 0 \% \\
\text { - No Grade } 4 \text { or } 5 \text { treatment-related events occurred } \\
\text { Subgroup results from the analysis were also reported for the type of } \\
\text { tumor (thyroid and salivary gland and GI tumors) } \\
\text { An additional } 19 \text { children and } 25 \text { adults (age range, } 0.1-78 \text { years) with } \\
\text { TRK fusion cancer were subsequently enrolled in the NCT02122913, } \\
\text { SCOUT and NAVIGATE trials after the primary analysis set. In the } 35 \\
\text { evaluable patients with a median follow-up of } 5.5 \text { months: } \\
\text { - Median DoR not been reached } \\
\text { - } 88 \% \text { of responses were ongoing at } 6 \text { months, consistent with the } \\
\text { primary analysis set (above) }\end{array}$ & {$[21,32]$} \\
\hline \multicolumn{5}{|l|}{ Entrectinib } \\
\hline $\begin{array}{l}\text { Pooled analysis of } \\
\text { ALKA-371-001 and } \\
\text { STARTRK-1 }\end{array}$ & $\begin{array}{l}\text { Cohort of patients with tumors } \\
\text { harboring } N T R K 1 / 2 / 3, R O S 1 \text { or } A L K \\
\text { gene fusions, naive to prior TKI } \\
\text { treatment targeting the specific } \\
\text { gene, and who were treated at } \\
\text { doses that achieved therapeutic } \\
\text { exposures consistent with the } \\
\text { recommended Phase II dose }\end{array}$ & $119(8)$ & $\begin{array}{l}\text { It is reported that in three patients with } N T R K 1 / 2 / 3 \text {-rearranged } \\
\text { advanced solid tumors with RECIST-measurable disease, the ORR was } \\
100 \%(95 \% \mathrm{Cl}: 44-100) \text {. }\end{array}$ & [26] \\
\hline $\begin{array}{l}\text { Pooled analysis of } \\
\text { ALKA-371-001, } \\
\text { STARTRK-1 and } \\
\text { STARTRK-2 }\end{array}$ & $\begin{array}{l}\text { Adult patients with } \\
\text { advanced/metastatic } \\
\text { NTRK-fusion-positive solid tumors } \\
\text { (ten tumor types, }>19 \\
\text { histopathology types), including } \\
\text { patients with baseline CNS } \\
\text { metastases }\end{array}$ & $54(54)$ & 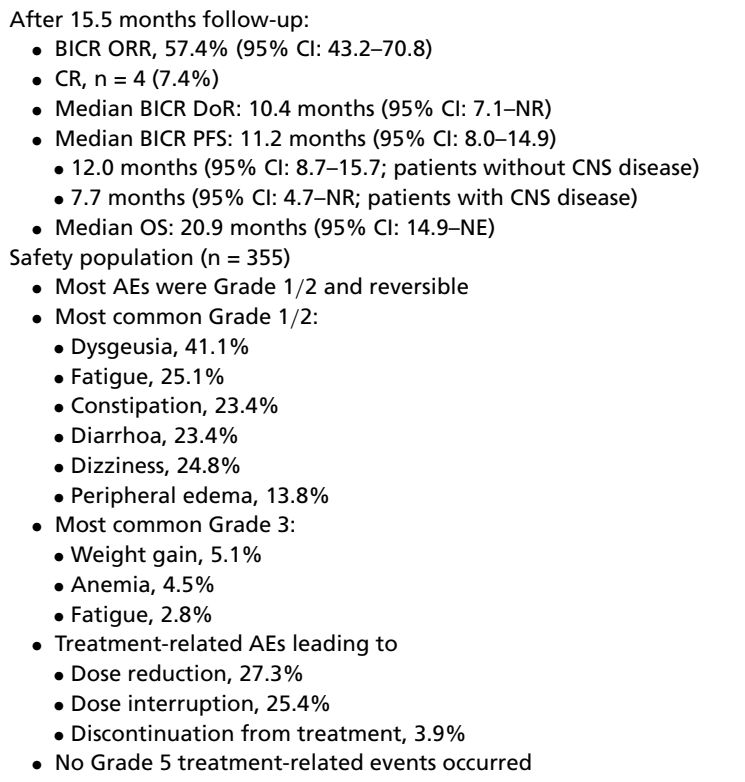 & [17] \\
\hline \multicolumn{5}{|c|}{$\begin{array}{l}\text { †Independent review. } \\
\text { †Investigator assessment. } \\
\text { AE: Adverse event; BICR: Blinded-independent central review; CR: Complete response; DoR: Duration of response; GI: Gastrointestinal; NE: Not evaluable; NR: Not reported; ORR: } \\
\text { Objective response rate; OS: Overall survival; PFS: Progression-free survival; RECIST: Response evaluation criteria in solid tumors. }\end{array}$} \\
\hline
\end{tabular}


fusion-positive solid tumors) with patients with $N T R K$-fusion-positive solid tumors representing a proportion (ranging from 2 to $71 \%$ ) of the total study populations. The results available across these individual studies for patients with NTRK fusion tumors are limited both in terms of end points reported and the number of patients included; a single patient (out of 41 patients in the trial; NCT02122913), six patients (NCT03025360) or 17 patients (out of 24 patients in the trial; SCOUT). However, a pooled analysis of patients with NTRK-fusionpositive tumors from the single-arm studies conducted in adult (NCT02122913), pediatric (SCOUT) and mixed adolescent and adult (NAVIGATE) populations has been reported across multiple publications $[21,32]$ and includes subgroup data for patients with a gastrointestinal malignancy [34] and for patients with thyroid and salivary gland cancers [40] (data shown in Supplementary information). The pooled analysis of the NCT02122913, SCOUT and NAVIGATE studies (Table 1) represents the most comprehensive dataset available for larotrectinib-treated tumor patients ( $\mathrm{n}=55$, including one patient with CNS metastases) and it reports an ORR of 75\% (95\% CI: 61-85) and 80\% (95\% CI: 67-90) as assessed by independent review and investigator assessment, respectively [21]. After 1 year, $71 \%$ of responses were on-going, $55 \%$ of patients remained progression free and $90 \%$ of patients were still alive. In a second phase of this pooled analysis, 44 patients were subsequently added after the primary analysis set and after 5.5 months although the median duration of response (DoR) has not been reached, $88 \%$ of responses were ongoing [32].

Larotrectinib was also well tolerated as adverse events (AEs) as clinically significant AEs were uncommon, with the majority (93\%) of all the AEs being of Grade 1 or 2 [21]. Few AEs of Grade 3 or 4, regardless of attribution, were observed. The most common were anemia (in 11\% of the patients), an increase in the ALT or AST level (in 7\%), weight increase (in 7\%) and a decrease in the neutrophil count (in 7\%). No Grade 4 or 5 events were considered by the investigators to be related to treatment, and no treatment-related Grade $3 \mathrm{AEs}$ occurred in more than $5 \%$ of the patients. Treatment-related AEs that lead to dose reduction occurred in $15 \%$ of patients and no patients who had a response discontinued larotrectinib because of an AE.

\section{Entrectinib}

Four trials were identified that reported the efficacy entrectinib in patients with NTRK-fusion-positive solid tumors: ALKA 372-001 (EudraCT 2012-000148-88; Phase I) [16,17,26], STARTRK-1 (NCT02097810; Phase I) [17,26,35], STARTRK-NG (NCT02650401; Phase 1) [18,19] and STARTRK-2 (NCT02097810; Phase I) [17,25]. All entrectinib studies included patients with multiple fusion-driven solid tumors (i.e., not limited to NTRK-fusion-positive solid tumors) with patients with $N T R K$-fusion-positive solid tumors representing a small proportion of the total study populations (ranging from 1.9\% in ALKA 372-001 [EudraCT 2012-000148-88] [16,17,26] to 25\% in STARTRK2 [NCT0209781] [17,25]; data shown in Supplementary information). The studies reported limited outcome data specifically for the subpopulation of patients with $N T R K$-fusion-positive solid tumors; however, a recently published abstract reported a pooled analysis of patients with $N T R K$-fusion-positive tumors from the single-arm studies conducted in adult populations identified as part of the systematic review (STARTRK-2, STARTRK-1 and ALKA-371-001) [17]. The pooled analysis provides outcome data for a total of 54 NTRK-fusion-positive tumor patients including 12 patients with CNS metastases receiving entrectinib who were followed-up for a duration of 15.5 months and this analysis represents the most comprehensive dataset available for entrectinib-treated tumor patients (Table 1) [17]. After 15.5 months of follow-up, an ORR of 57.4\% (95\% CI: 43.2-70.8) assessed by blinded independent central review (BICR) was reported and a complete response (CR) was observed in four patients (7.4\%). The median DoR (by BICR) was 10.4 months (95\% CI: 7.1-NR) and the median OS was 20.9 months (95\% CI: 14.9-not reported). The overall median PFS (as assessed by BICR) was 11.2 months (95\% CI: 8.0-14.9), which was longer than in patients with CNS disease (7.7 months [95\% CI: 4.7-NR]), but slightly less than patients without CNS disease (12.0 months [95\% CI: 8.7-15.7]).

In the pooled analyses of the entrectinib trials, the overall safety population consisted of 355 patients with the $N T R K$-fusion-positive safety population including 68 patients [17]. Most AEs were Grade $1 / 2$ and reversible with the most common being dysgeusia ( $41.1 \%$ in the overall and $47.1 \%$ in the NTRK-fusion-positive population), followed by fatigue (25.1 and 27.9\%), constipation (23.4 and 27.9\%), diarrhea (23.4 and 27.9\%), dizziness (24.8 and 23.5\%) and peripheral edema (13.8 and 23.5\%) in the overall and NTRK-fusion-positive population, respectively. The most common Grade 3 AEs were weight gain $5.1 \%$ in the overall and $10.3 \%$ in the NTRKfusion-positive population), followed by anemia (4.5 and 11.8\%) and fatigue (2.8 and 7.4\%) in the overall and $N T R K$-fusion-positive population, respectively. In the overall safety population treatment-related AEs that lead to dose reduction occurred in $27.3 \%$, dose interruption in $25.4 \%$ and discontinuation from treatment occurred in 
$3.9 \%$ of patients. No Grade 5 treatment-related events were observed. Treatment-related AEs in the NTRK-fusionpositive safety population were consistent with the overall safety population.

\section{Repotrectinib}

Clinical data relating to repotrectinib were reported in a single clinical study in patients with advanced solid tumors harboring $A L K, R O S 1$ or NTRK1-3 rearrangements (NCT03093116; TRIDENT-1). The trial enrolled seven evaluable $N T R K$-fusion-positive patients, but outcome data were limited and generally related to single patients [24].

\section{Current evidence for other treatments}

Data on other treatments were limited. Two publications reported the baseline characteristics and study design of a Phase I/IIa open-label trial assessing the efficacy and safety of oral belizatinib in patients with advanced solid tumors and lymphomas (NCT02048488) [30,33]. Preliminary dose-finding results from these studies were published as an abstract in 2014 [36] and an update on the enrolled patients with an $A L K^{+}$mutation was published in 2015 [42]. One publication reported on the efficacy of LOXO-195 in two patients with TRK-fusion-positive cancers who developed acquired resistance mutations to larotrectinib. Treatment with LOXO-195 led to rapid tumor responses and extended the overall duration of disease control achieved with TRK inhibition in both patients [22]. A single publication reported results from a trial on the efficacy of cabozantinib (NCT01639508) with participants divided into three groups based on their gene fusion: tumors with a RET fusion; tumors with an NTRK fusion, or MET or $A X L$ overexpression, amplification or mutation; and tumors with a ROS1 fusion. Only data from patients whose tumors have a RET fusion have been published to date [19]. Preliminary data from a Phase Ia clinical trial assessing sitravatinib (MGCD516) in patients with non-small-cell lung cancer with genetic alterations in MET, AXL, RET, TRK, DDR2, KDR, PDGFRA, KIT or CBL (NCT02219711) indicate favorable pharmacokinetic characteristics, on-target pharmacodynamic effects and treatment was associated primarily with constitutional or GI-related AEs [14].

A number of ongoing early-phase trials using targeted agents for the treatment of NTRK-fusion-positive tumors were identified through searching of clinical trial websites, and although preclinical results are available, there are as yet no published reports of trial efficacy or safety. Merestinib (LY2801653) is being evaluated in patients with advanced non-small-cell lung cancer with a $M E T$ exon 14 mutation or patients with advanced cancer harboring an NTRK1/2/3 rearrangement in a trial that is expected to complete by March 2024 (NCT02920996). Two clinical trials were identified that examined the safety and efficacy of DS-6051b in patients with advanced solid malignant tumors harboring either a ROS1 or NTRK fusion gene (NCT02279433 and NCT02675491), which were estimated to complete by March 2019 and November 2019, respectively. The safety and pharmacokinetics of PLX7486 was assessed in a Phase I, open-label study in patients with solid tumors harboring NTRK-fusion mutations, who reported tumor progression following standard therapy, had treatment-refractory disease, or for whom there was no effective standard therapy (NCT01804530). This trial was expected to complete in early 2018, however, no results have been published to date.

\section{Quantitative analysis of clinical data}

On completion of the systematic review, a meta-analysis feasibility assessment was conducted to ascertain whether a robust comparison of relative treatment effects for TRK-targeted agents for outcomes of interest and restricted to specific tumor types was possible. The assessment of single-arm studies relies on naive indirect comparisons and it is generally recommended that such comparisons are avoided due to the susceptibility of bias [43]. In the current project, naive indirect comparisons were technically 'feasible' using data from the published pooled analyses for entrectinib and larotrectinib for the outcome of ORR only $[17,21,32]$. However, the data for entrectinib [17] were based on adult populations versus the data for larotrectinib [21,32], which were based on a mixed pediatric and adult population and there were substantial differences in follow-up period between the analyses (15.5 months vs 1 year, respectively). An unanchored matching adjusted indirect comparison analysis was not feasible for specific tumor types due to the small-sample size of the available entrectinib (54 patients across ten different tumor types, 12 patients with CNS metastases) and larotrectinib (55 patients across 12 different tumor types, one patient with CNS metastases) datasets (Table 2). After completion of the full feasibility assessment, it was concluded that a robust meta-analysis comparing entrectinib and larotrectinib was not feasible due to small-sample size and heterogeneity between the patient populations. 
Table 2. Baseline characteristics of patients with NTRK gene fusions from pooled analyses for larotrectinib and entrectinib clinical trials.

\begin{tabular}{|c|c|c|c|c|c|c|c|c|}
\hline Study & $\begin{array}{l}\text { No. with } \\
\text { NTRK fusion }\end{array}$ & $\begin{array}{l}\text { Age, years, } \\
\text { median } \\
\text { (range) }\end{array}$ & $\begin{array}{l}\text { Sex, } \\
\text { male/female }\end{array}$ & $\begin{array}{l}\text { ECOG } \\
\text { performance } \\
\text { status, n (\%) }\end{array}$ & $\begin{array}{l}\text { Prior } \\
\text { systemic } \\
\text { therapies, } \mathrm{n} \\
(\%)\end{array}$ & $\begin{array}{l}\text { No. with CNS } \\
\text { metastases }\end{array}$ & Tumor type, n (\%) & Ref. \\
\hline \multicolumn{9}{|l|}{ Larotrectinib } \\
\hline $\begin{array}{l}\text { Pooled analysis } \\
\text { (NCT02122913, SCOUT and } \\
\text { NAVIGATE) }\end{array}$ & 55 & $45(0.3-76.0)$ & $29 / 26$ & $\begin{array}{l}0,24(44 \%) \\
1,27(49 \%) \\
2,4(7 \%) \\
\text { Unknown, } 0\end{array}$ & $\begin{array}{l}0-1,27 \\
(49 \%) \\
2,9(16 \%) \\
\geq 3,19(35 \%)\end{array}$ & 1 & $\begin{array}{l}\text { Salivary gland, } 12(22 \%) \\
\text { Soft tissue sarcoma, } 11(20 \%) \\
\text { Infantile fibrosarcoma, } 7(13 \%) \\
\text { Thyroid, } 5(9 \%) \\
\text { Colon, } 4(7 \%) \\
\text { Lung, } 4(7 \%) \\
\text { Melanoma, } 4(7 \%) \\
\text { GIST, } 3(5 \%) \\
\text { Cholangiocarcinoma, } 2(4 \%) \\
\text { Appendix, } 1(2 \%) \\
\text { Breast, } 1(2 \%) \\
\text { Pancreas, } 1(2 \%)\end{array}$ & {$[21,32]$} \\
\hline \multicolumn{9}{|l|}{ Entrectinib } \\
\hline $\begin{array}{l}\text { Pooled analysis of } \\
\text { ALKA-371-001, STARTRK-1 } \\
\text { and STARTRK-2 }\end{array}$ & 54 & $57.5(21-83)$ & $22 / 32$ & NR & $\begin{array}{l}0,20(37 \%) \\
1,11(20.4 \%) \\
\geq 2,23 \\
(42.6 \%)\end{array}$ & 12 & $\begin{array}{l}\text { Soft tissue sarcoma, } 13(24 \%) \\
\text { Thyroid, } 5(9 \%) \\
\text { Colon, } 4(7 \%) \\
\text { Lung, } 10(19 \%) \\
\text { Cholangiocarcinoma, } 1(2 \%) \\
\text { Breast, } 6(11 \%) \\
\text { Pancreas, } 3(6 \%) \\
\text { Neuroendocrine, } 3(6 \%) \\
\text { Gynaecological, } 2(4 \%) \\
\text { MASC, } 7(13 \%)\end{array}$ & [17] \\
\hline
\end{tabular}

Economic evaluation, QoL \& cost/resource use studies

No publications were identified that reported economic evaluations or QoL or cost/resource use data in patients with $N T R K$-fusion-positive solid tumors. Although a small number of conference abstracts were identified, which discussed the economics of genetic tests (e.g., next-generation sequencing or sequential single-gene testing) to detect genomic alterations in patients, such studies were not focused specifically on the detection of NTRK mutations, therefore, did not meet the criteria for inclusion in the systematic review $[44,45]$.

\section{Discussion}

This systematic review identified a general paucity of data regarding TRK-targeted agents. Despite a total of 19 single-arm clinical studies examining ten unique interventions to treat patients with NTRK-fusion-positive tumors, clinical data were only available for three interventions: entrectinib, larotrectinib and repotrectinib [15-38] and no evidence was identified for economic evaluation, QoL and cost/resource use outcomes for these tumors. Clinical research in this indication is in its early stages and much of the data on clinical efficacy and safety have been reported in single-patient case reports [46-64]. However, despite a lack of data, the initial results for TRK -targeted agents have been encouraging with agents showing promising efficacy and favorable safety profiles in Phase I trials although these data require further corroboration from an expansion of the current published evidence base and the addition of 'real word' data as observed in routine clinical practice. The paucity of published economic evaluations, cost/resource use data or QoL specific to the NTRK indication is not surprising, primarily due to the low prevalence of the disease.

Quantitative synthesis remains a challenge in a tumor-agnostic setting. As NTRK fusions are only expressed in up to $1 \%$ of all solid tumors $[2,3]$, there are inherent difficulties in assessing the efficacy of interventions in the rare tumor types, which exhibit this genomic alteration at a high frequency as was observed in the current review. Patient numbers enrolled in trials are low, the ongoing trials are single-arm, open-label studies and the study population is heterogenous with regard to baseline characteristics (e.g., age, ECOG status and tumor site, presence of CNS metastases). Therefore, a robust quantitative synthesis of results comparing the relative efficacy of the different $N T R K$ mutation-specific agents versus the current standards of care is challenging. 
Although the available clinical evidence to date suggests a pivotal role for TRK-targeted agents in selected patients with solid tumors, there are several issues associated with their use which need to be considered. There is evidence in some patients that $N T R K$-TKI treatment has resulted in an acquired resistance [65], as has been observed in EGFR mutant, $A L K$ - or ROS1-fusion-positive lung cancer [66-68]. Two cases of entrectinib resistance [49,57] and nine cases of an acquired resistance to larotrectinib have been reported [21]. In vitro studies have reported that experimental drugs such as ponatinib and nintedanib effectively inhibited the survival of specific NTRK mutants, which showed resistance to entrectinib or larotrectinib [65] and in the clinic, two next-generation TRK inhibitors (LOXO-195 and repotrectinib) are being tested to overcome these recurrent resistance mutations [22,30,69-71]. A recent Phase I study investigating the effects of LOXO-195 on patients with larotrectinib-resistant NTRK-fusion-positive tumors showed that of the 29 patients who were evaluable for response, ten (34\%) had a confirmed CR or partial response. When the response rates were analyzed by the mechanism of underlying resistance to the first-generation TRK inhibitors, it was reported that 9 of 20 patients (45\%) whose tumors had become resistant by acquiring an NTRK gene mutation had a CR or partial response. None of the three patients whose tumors had become resistant by TRK-independent mechanisms responded to LOXO-195 [70].

The development of tumor-agnostic therapies brings new challenges when seeking payer reimbursement. In the current regulatory landscape, due to the lack of experience and precedent, neither regulators nor researchers or sponsors know precisely what is required for approval of new agents based solely on presence and response of a molecular target [72]. This may require further methodological development for regulatory acceptance [73]. Encouraging data from the clinical trial programs have recently culminated in the approval of the first 'tumoragnostic' TKI agents (larotrectinib and entrectinib) by the FDA for patients with solid tumors that have an NTRK gene fusion despite there being no FDA-approved test for NTRK fusions $[8,9,74]$. However, there may be additional challenges when seeking approval from more traditional HTA agencies where current oncology appraisal pathways are structured based on treating tumors from a single origin site. Fitting the evidence to the HTA framework in these situations is difficult. Although in the UK the NICE has previously made recommendations based on single-arm trials [75], NICE and most other HTA agencies have a preference for evidence generated from RCTs, which are considered to be the 'gold-standard' design for assessing treatment effectiveness [76]. However, conducting adequately powered RCTs as part of the development of treatments for rare mutations such as NTRK is not feasible; consequently basket trials are more commonplace [77]. Challenges are also present when defining an active comparator. Most HTA agencies require that the new technology is assessed against the standard of care for that indication. In cases with tumor-agnostic therapies, multiple standards of care are available depending on the tumor location. In addition, traditional accepted end points in oncology such as OS and PFS (as are preferred by HTA bodies) may not be available for rarer diseases and a lack of certainty of how particular biomarker-based end points link to these outcomes may devalue the level of submitted evidence.

Screening procedures to identify gene fusions are costly to apply to all patients and it is uncertain whether HTA agencies should consider the cost of the diagnostic testing as part of the overall treatment cost for the drug as part of any reimbursement assessment. In July 2019, the European Society of Medical Oncology (ESMO) published recommendations on standard methodology to detect NTRK fusions in daily practice and clinical research [78]. It is recommended that fluorescence in situ hybridization or reverse transcription-PCR (RT-PCR) should be used in tumors where NTRK fusions are highly recurrent compared with assays allowing fusion gene detection in an agnostic manner where a limited proportion of cases are expected to harbor NTRK fusions. As more tumor-agnostic therapies become available in the future, it will be imperative that HTA bodies consider any additional diagnostic costs in light of improvements in clinical efficacy reported for these agents in the management of patients where there is currently a substantial unmet need.

Other challenges lie in the HTA assessment of the cost, economic valuation and quality of life evidence. In such cases, there is liable to be a lack of data and in a traditional oncology reimbursement the indication may be widened (i.e., the costs/QoL of treating a less specific cancer type in that site would be considered). However, this is not possible for tumor-agnostic tumors as locations vary between patients. Often, there are no standard protocols within the HTA submission processes to deal with these issues. In England, however, the Cancer Drugs Fund exists as an interim funding option for cancer medicines whose cost-effectiveness is subject to a higher than normal degree of uncertainty; during the interim funding period, data collection is usually mandated to help address this uncertainty. The Cancer Drugs Fund is, therefore, a potential option for tumor-agnostic therapies, since it provides a framework with which to address key areas of clinical and economic uncertainty [79]. In addition, NICE also has a 
highly specialized technology pathway, with adjusted thresholds and evidence requirements, although the pathway is limited at the moment to single indications in very rare conditions, and it has yet to consider oncology drugs [75].

To our knowledge, this is the first systematic review that assessed the efficacy and safety of TKIs in patients with NTRK fusions. However, the study had some limitations. First, as previously mentioned due to the rare nature of the indication, low patient numbers, single-arm trials and heterogeneous populations made it difficult to make robust comparisons between interventions. Second, only two full primary publications containing efficacy data were included [27,31]. Apart from one full publication containing pooled evidence for several trials [21], the remaining data were extracted from conference abstracts and due to their brevity, it was not possible to assess the quality of the study or the reporting. There was also a lack of access to individual patient data and data on AEs were missing for several reports.

\section{Conclusion}

Overall, this review identified several tumor-agnostic treatments that target cancers with an NTRK gene fusion that are being studied in ongoing clinical studies. So far, the clinical response reported to date is impressive. Such treatments may provide a molecular basis for personalized therapy in tumor types that have traditionally been considered prognostically poor and therefore the further development of such TKI agents should be considered a priority in cancer research.

\section{Future perspective}

Although data demonstrate that TRK-targeted agents are clinically beneficial and are well tolerated in hard-to-treat $N T R K$-fusion-positive solid tumors, challenges remain in their integration into routine clinical practice. Further clinical data are required to enable quantitative comparison of the relative efficacy of agents for clinically meaningful outcomes. Due to the rarity of these mutations, it will take time to generate a substantial robust dataset, therefore data will likely come from a real-world evidence base. Research is also required to address the potential challenge of treatment resistance, although preliminary studies have shown encouraging results in overcoming resistance through the use of next-generation TRK inhibitors (LOXO-195 and repotrectinib). Following the recent FDA approvals of larotrectinib and entrectinib, progress is required in the validation and establishment of large-scale, readily accessible, cost-effective pretreatment testing algorithms to identify suitable patients. Current hurdles when seeking HTA reimbursement for tumor-agnostic therapies must also be overcome, including the acceptance of data from single-arm trials to demonstrate the efficacy and safety of potential tumor-agnostic therapies, and how to account for substantial testing costs in economic modeling without penalizing first movers. Incorporation of the TRK-targeted agents into routine clinical practice would permit a paradigm shift in the management of patients, identified through routine biomarker testing, based on the genetic makeup of their tumor and not by anatomical location.

Supplementary data

To view the supplementary data that accompany this paper please visit the journal website at: www.futuremedicine.com/doi/suppl/10.2217/fon-2019-0534

\section{Author contributions}

All the authors, including the authors employed by the sponsor (M Hodgson, A Steenrod and P Chu), participated in the development of the research question. The literature searches and screening of retrieved articles were conducted by CR Mitchell and $S$ Batson of Mtech Access Ltd. (Bicester, UK). All the authors were involved in the analysis and interpretation of the data and in the development of the manuscript. The authors did not receive any compensation for the writing of the manuscript. The decision to submit for publication was that of the authors alone, and all the authors were involved in this decision. All the authors have approved the final version of the article, including the authorship list.

Financial \& competing interests disclosure

M Hodgson, A Steenrod and P Chu are employees of Roche Ltd. CR Mitchell and S Batson are employees of Mtech Access Ltd., which received payment from Roche Ltd. to undertake the literature review. The authors have no other relevant affiliations or financial involvement with any organization or entity with a financial interest in or financial conflict with the subject matter or materials discussed in the manuscript apart from those disclosed. This work was supported by F. Hoffmann-La Roche Ltd. (Basel, 
Switzerland). The authors have no other relevant affiliations or financial involvement with any organization or entity with a financial interest in or financial conflict with the subject matter or materials discussed in the manuscript apart from those disclosed.

This manuscript was written by the authors. The literature review and writing of the manuscript was supported by F. HoffmannLa Roche Ltd. (Basel, Switzerland).

\section{Ethical conduct of research}

No ethical approval was required for this research, however the systematic review was conducted using the PRISMA guidelines (http://www.prisma-statement.org/).

\section{Open access}

This work is licensed under the Attribution-NonCommercial-NoDerivatives 4.0 Unported License. To view a copy of this license, visit http://creativecommons.org/licenses/by-nc-nd/4.0/

\section{Summary points}

\section{Background}

- Neurotrophic tropomyosin-related receptor tyrosine kinases (NTRK) mutations occur in up to $1 \%$ of all solid tumors and are observed in more than 20 different malignancies over diverse tissue/cell lineages. The clinical effectiveness of TRK inhibitors is being assessed in Phase I and II trials.

- A systematic review (SR) was conducted to provide a summary of published evidence for the currently available TRK-targeted agents and a meta-analysis feasibility was also conducted to assess whether quantitative comparisons could be made on their relative efficacy and safety.

Methods

- The electronic databases Medline ${ }^{\circledR}$, Medline ${ }^{\circledR}$ Epub Ahead of Print (In-Process \& Other Non-Indexed Citations), Embase and EBM Reviews were interrogated.

- Eligibility criteria included clinical studies, economic evaluations, utility studies or cost/resource use studies conducted in patients (adults or pediatric patients) with NTRK-fusion-positive solid tumors.

\section{Results of the SR}

- A total of 27 relevant clinical studies associated with seven TRK-targeted agents were eligible for inclusion in the SR.

- However, clinical efficacy/safety data were only available for two interventions: entrectinib and larotrectinib.

- Data from pooled analyses demonstrate that both larotrectinib and entrectinib are effective in treating tumors-harboring NTRK-fusion-positive mutations and are well tolerated across multiple tumor types.

- No relevant economic or quality of life studies were identified.

Meta-analysis feasibility

- A meta-analysis feasibility assessment to compare the relative efficacy of entrectinib and larotrectinib for outcomes of interest.

- However, a robust meta-analysis was not feasible due to the single-arm design of eligible studies, heterogeneity of outcome data and small sample sizes.

\section{Conclusion}

- Preliminary data clearly demonstrate the currently available TRK-targeted agents to be generally well tolerated and associated with an impressive clinical benefit (as assessed by objective response rate).

- Further corroboration from the expansion of existing studies and real-world data is required to further elucidate the optimum positioning of TRK-targeted agents in the treatment pathway.

\section{References}

Papers of special note have been highlighted as: $\bullet$ of interest $\bullet \bullet$ of considerable interest

1. Borset M. Tumour-agnostic drugs and future cancer treatment. Tidsskr. Nor Laegeforen. 139(5), (2019).

2. Cocco E, Scaltriti M, Drilon A. NTRK fusion-positive cancers and TRK inhibitor therapy. Nat. Rev. Clin. Oncol. 15, 731-747 (2018).

3. Gatalica Z, Xiu J, Swensen J et al. Molecular characterization of cancers with NTRK gene fusions. Mod. Pathol. 32, 147-153 (2019).

4. Lange AM, Lo HW. Inhibiting TRK proteins in clinical cancer therapy. Cancers doi:10.3390/cancers10040105, 10 (2018).

5. Chen Y, Chi P. Basket trial of TRK inhibitors demonstrates efficacy in TRK fusion-positive cancers. J. Hematol. Oncol. 11, 78 (2018).

6. Kummar S, Lassen UN. TRK inhibition: a new tumor-agnostic treatment strategy. Target. Oncol. 13, 545-556 (2018).

7. Ricciuti B, Brambilla M, Metro G et al. Targeting NTRK fusion in non-small cell lung cancer: rationale and clinical evidence. Med. Oncol. 34, 105 (2017). 
8. USFDA. FDA approves larotrectinib for solid tumors with $N T R K$ gene fusions (2018). www.fda.gov/Drugs/InformationOnDrugs/ApprovedDrugs/ucm626720.htm

9. USFDA. FDA approves third oncology drug that targets a key genetic driver of cancer, rather than a specific type of tumor (2019). www.fda.gov/news-events/press-announcements/fda-approves-third-oncology-drug-targets-key-genetic-driver-cancer-rather-specific- $t$ ype-tumor

10. Laetsch TW, Hawkins DS. Larotrectinib for the treatment of TRK fusion solid tumors. Expert Rev. Anticancer Ther. 19, 1-10 (2018).

11. Moher D, Shamseer L, Clarke M et al. Preferred reporting items for systematic review and meta-analysis protocols (PRISMA-P) 2015 statement. Syst Rev 4, 1 (2015).

12. Centre for Reviews and Dissemination. Systematic reviews: CRD's guidence for undertaking reviews in health care. University of York York, UK, 2- 281 (2009).

13. National Institute for Health and Care Excellence. Quality appraisal checklist - quantitative intervention studies (2012). https://www.nice.org.uk/process/pmg4/chapter/appendix-f-quality-appraisal-checklist-quantitative-intervention-studies

14. Bauer T, Adkins D, Schwartz GK et al. A first in human Phase I study of receptor tyrosine kinase (RTK) inhibitor MGCD516 in patients with advanced solid tumors. J. Clin. Oncol. 34(15), 2575 (2016).

15. Burris HA, Brose MS, Shaw A et al. A first-in-human study of LOXO-101, a highly selective inhibitor of the tropomyosin receptor kinase (TRK) family. J. Clin. Oncol. 33(15), 2624 (2017).

16. De Braud FG, Niger M, Damian S et al. Alka-372-001: first-in-human, Phase I study of entrectinib - an oral pan-trk, ROS1, and ALK inhibitor - in patients with advanced solid tumors with relevant molecular alterations. J. Clin. Oncol. 33(15), 2517 (2017).

17. Demetri GD, Paz-Ares L, Farago A et al. Efficacy and safety of entrectinib in patients with NTRK fusion-positive tumours: pooled analysis of STARTRK-2, STARTRK-1, and ALKA-372- 001. Ann. Oncol. 29(9), ix175 (2018).

-• Publication represents the most comprehensive dataset of the efficacy and safety of entrectinib in patients with $N T R K$-fusion-positive tumors.

18. Desai AV, Brodeur GM, Foster J et al. Phase 1 study of entrectinib (RXDX-101), a TRK, ROS1, and ALK inhibitor, in children, adolescents, and young adults with recurrent or refractory solid tumors. J. Clin. Oncol. 36(15), 10536 (2018).

19. Desai AV, Brodeur GM, Foster J et al. STARTRK-NG: a Phase I/Ib study of entrectinib in children and adolescents with advanced solid tumors and primary CNS tumors, with or without TRK, ROS1, or ALK fusions. Proceedings: AACR Annual Meeting 2017. Washington, DC, USA, 1-5 April 2017.

20. Drilon A, Hong DS, Deeken J et al. A Phase II basket study of the oral TRK inhibitor LOXO-101 in adult subjects with NTRK fusion-positive tumors. Ann. Oncol. 34(15), 2599 (2016).

21. Drilon A, Laetsch TW, Kummar S et al. Efficacy of larotrectinib in TRK fusion-positive cancers in adults and children. N. Engl. J. Med. 378, 731-739 (2018).

-. Publication represents the most comprehensive dataset of the efficacy and safety of larotrectinib in patients with NTRK-fusion-positive tumors.

22. Drilon A, Nagasubramanian R, Blake J et al. A next-generation TRK kinase inhibitor overcomes acquired resistance to prior TRK kinase inhibition in patients with TRK fusion-positive solid tumors. Cancer Discov. 7, 963-972 (2017).

23. Drilon A, Rekhtman N, Arcila M et al. Cabozantinib in patients with advanced RET-rearranged non-small-cell lung cancer: an open-label, single-centre, Phase II, single-arm trial. Lancet Oncol. 17, 1653-1660 (2016).

24. Drilon A, Ou SH, Cho BC et al. A Phase I study of the next-generation ALK/ROS1/TRK inhibitor ropotrectinib (TPX-0005) in patients with advanced ALK/ROS1/NTRK+ cancers (TRIDENT-1). J. Clin. Oncol. 36(15), 2513 (2018).

25. Drilon A, Sankhala KK, Liu SV et al. STARTRK-2: a global Phase II, open-label, basket study of entrectinib in patients with locally advanced or metastatic solid tumors harboring TRK, ROS1, or ALK gene fusions. Proceedings: AACR Annual Meeting 2017. Washington, DC, USA, 1-5 April 2017.

26. Drilon A, Siena S, Ou SI et al. Safety and antitumor activity of the multitargeted Pan-TRK, ROS1, and ALK inhibitor entrectinib: combined results from two Phase I trials (ALKA-372-001 and STARTRK-1). Cancer Discov. 7, 400-409 (2017).

-. Publication represents a pooled analysis of two trials assessing the efficacy and safety of entrectinib in patients with $N T R K$-fusion-positive tumors.

27. DuBois SG, Laetsch TW, Federman N et al. The use of neoadjuvant larotrectinib in the management of children with locally advanced TRK fusion sarcomas. Cancer 124, 4241-4247 (2018).

28. Gallego Melcon S, Casanova M, Bielack S et al. Phase $1 / 2$ study of the selective TRK inhibitor larotrectinib, in pediatric patients with cancer. Ann. Oncol. 28, v139 (2017).

29. Hong DS, Farago A, Brose MS et al. Clinical safety and activity from a Phase 1 study of LOXO-101, a selective TRKA/B/C inhibitor, in solid-tumor patients with NTRK gene fusion. Ann. Oncol. 27(9), ix46-ix51 (2016).

30. Hyman DM, Laetsch TW, Kummar S et al. The efficacy of larotrectinib (LOXO-101), a selective tropomyosin receptor kinase (TRK) inhibitor, in adult and pediatric TRK fusion cancer. J. Clin. Oncol. 35(18), 2501 (2017). 
31. Laetsch TW, DuBois SG, Mascarenhas L et al. Larotrectinib for paediatric solid tumours harbouring NTRK gene fusions: Phase I results from a multicentre, open-label, Phase I/II study. Lancet Oncol. 19, 705-714 (2018).

32. Lassen UN, Albert CM, Kummar S et al. Larotrectinib efficacy and safety in TRK fusion cancer: an expanded clinical dataset showing consistency in an age and tumor agnostic approach. Ann. Oncol. 29(8), viii133-viii148 (2018).

-. Publication reports the most recent (as of date cutoff for the current review in October 2018) data from the pooled analysis of larotrectinib trials.

33. Nagasubramanian R, DuBois SG, Laetsch TW et al. A pediatric Phase I study of LOXO-101, a highly selective inhibitor of the tropomyosin receptor kinase (TRK) family. J. Clin. Oncol. 34(15), 10583 (2016).

34. Nathenson M, Demetri GD, Lassen UN et al. Activity of larotrectinib in patients with TRK fusion GI malignancies. Ann. Oncol. 29(5), v107 (2018).

35. Patel MR, Bauer TM, Liu SV et al. STARTRK-1: Phase I/IIa study of entrectinib, an oral Pan-Trk, ROS1, and ALK inhibitor, in patients with advanced solid tumors with relevant molecular alterations. J. Clin. Oncol. 33(Suppl.), Abstr 2596 (2015).

36. Sachdev JC, Arkenau HT, Infante JR et al. Phase (Ph) I/IIa study of TSR-011, a potent inhibitor of ALK and TRK, in advanced solid tumors including crizotinib-resistant ALK positive non-small cell lung cancer. Eur. J. Cancer 50, 165 (2014).

37. Suehara Y, Trahair T, Kirby M et al. Expanded access of larotrectinib to manage pediatric trk fusion sarcomas. Pediatr. Blood Cancer 65, S335-S336 (2018).

38. Turpin B, Albert CM, Mascarenhas L et al. A pediatric Phase I study of larotrectinib, a highly selective inhibitor of the tropomyosin receptor kinase (TRK) family: an updated analysis. Cancer Res. 78(19), Abstract nr PR07 (2018).

39. Weiss GJ, Sachdev JC, Infante JR et al. TSR-011: a potent inhibitor of ALK including crizotinib-resistant mutations in Phase I-II development for ALK+ NSCLC. J. Thor. Oncol. 8(11 S2), S618 (2013).

40. Wirth L, Drilon A, Albert CM et al. Larotrectinib is highly active in patients with advanced recurrent TRK fusion thyroid (TC) and salivary gland cancers (SGC). Int. J. Radiat. Oncol. 100, 1318 (2018).

41. Hong DS, Bauer TM, Lee JJ et al. Larotrectinib in adult patients with solid tumours: a multi-centre, open-label, Phase I dose-escalation study. Ann. Oncol. 30, 325-331 (2019).

42. Arkenau HT, Sachdev JC, Mita MM et al. Phase (Ph) I/Ila study of TSR-011, a potent inhibitor of ALK and TRK, in advanced solid tumors including crizotinib-resistant ALK positive non-small cell lung cancer. J. Clin. Oncol. 33, 8063-8063 (2015).

43. Glenny AM, Altman DG, Song F et al. Indirect comparisons of competing interventions. Health Technol. Assess. 9, 1-134 iii-iv (2005).

44. Layfield L, Hammer R, Schmidt R. Utilization of next generation sequencing vs. sequential individual testing for therapeutically important mutations in lung cancer: a cost effectiveness study. Mod. Pathol. 31, 738-739 (2018).

45. Pennell NA, Mutebi A, Zhou ZY et al. Economic impact of next generation sequencing vs sequential single-gene testing modalities to detect genomic alterations in metastatic non-small cell lung cancer using a decision analytic model. J. Clin. Oncol. 36, 9031-9031 (2018).

46. Alvarez-Breckenridge C, Miller JJ, Nayyar $\mathrm{N}$ et al. Clinical and radiographic response following targeting of BCAN-NTRK1 fusion in glioneuronal tumor. NPJ Precis Oncol. 1, 5 (2017).

47. Brastianos PK. Identification of novel NTRK fusion in glioneuronal tumours and radiographic response following therapy with an NTRK inhibitor. Neuro-Oncology 19, iii11 (2017).

48. Doebele RC, Davis LE, Vaishnavi A et al. An oncogenic NTRK fusion in a patient with soft-tissue sarcoma with response to the tropomyosin-related kinase inhibitor LOXO-101. Cancer Discov. 5, 1049-1057 (2015).

49. Drilon A, Li G, Dogan S et al. What hides behind the MASC: clinical response and acquired resistance to entrectinib after ETV6-NTRK3 identification in a mammary analogue secretory carcinoma (MASC). Ann. Oncol. 27, 920-926 (2016).

50. Farago AF, Le LP, Zheng Z et al. Durable clinical response to entrectinib in NTRK1-rearranged non-small cell lung cancer. J. Thorac. Oncol. 10, 1670-1674 (2015).

51. Felker J. Successful treatment of a secondary pediatric high grade glioma with a novel BEND4-NTRK2 fusion with entrectinib, a TRK inhibitor. Neuro-Oncology 20, i67 (2018).

52. Halalsheh $\mathrm{H}, \mathrm{McC}$ arville MB, Neel M et al. Dramatic bone remodeling following larotrectinib administration for bone metastasis in a patient with TRK fusion congenital mesoblastic nephroma. Pediatr. Blood Cancer 65, e27271 (2018).

53. Ho AL. Overcoming drug resistance to Trk inhibition by rational combination of entrectinib and trametinib: from bench to bedside. Eur. J. Cancer 69, S5-S6 (2016).

54. Landman Y, Ilouze M, Wein $S$ et al. Rapid response to larotrectinib (LOXO-101) in an adult chemotherapy-naive patients with advanced triple-negative secretory breast cancer expressing ETV6-NTRK3 fusion. Clin. Breast Cancer 18, e267-e270 (2018).

55. Nagasubramanian R, Wei J, Gordon P et al. Infantile fibrosarcoma with NTRK3-ETV6 fusion successfully treated with the tropomyosin-related kinase inhibitor LOXO-101. Pediatr. Blood Cancer 63, 1468-1470 (2016).

56. Rangaraju S. Pediatric Phase I/IB study of entrectinib in patients with primary brain tumours, neuroblastoma, and NTRK, ROS1, or ALK fusions. Neuro-Oncology 19, iv53 (2017).

57. Russo M, Misale S, Wei G et al. Acquired resistance to the TRK inhibitor entrectinib in colorectal cancer. Cancer Discov. 6, 36-44 (2016). 
58. Sartore-Bianchi A, Amatu A, Bonazzina E et al. Pooled analysis of clinical outcome of patients with chemorefractory metastatic colorectal cancer treated within Phase I/II clinical studies based on individual biomarkers of susceptibility: a single-institution experience. Target. Oncol. 12, 525-533 (2017).

59. Sartore-Bianchi A, Ardini E, Bosotti R et al. Sensitivity to entrectinib associated with a novel LMNA-NTRK1 gene fusion in metastatic colorectal cancer. J. Natl Cancer Inst. 108(1), 1-4 (2016).

60. Schram AM. Potential role of larotrectinib (LOXO-101), a selective pan-TRK inhibitor, in NTRK fusion-positive recurrent glioblastoma. Cancer Res. 77(143), Abstract LB-302 (2017).

61. Shukla N, Roberts SS, Baki MO et al. Successful targeted therapy of refractory pediatric ETV6-NTRK3 fusion-positive secretory breast carcinoma. JCO Precis Oncol. 1 2017, 1-8 (2017).

62. Sigal $\mathrm{D}$, Tartar M, Xavier $\mathrm{M}$ et al. Activity of entrectinib in a patient with the first reported NTRK fusion in neuroendocrine cancer. $J$. Natl Compr. Canc. Netw. 15, 1317-1322 (2017).

63. Wei G. Overcoming drug resistance to Trk inhibition by rational combination of entrectinib and trametinib: from bench to bedside. Mol. Cancer Ther. 16(10), Abstract B28 (2017).

64. Ziegler DS, Wong M, Mayoh C et al. Brief Report: potent clinical and radiological response to larotrectinib in TRK fusion-driven high-grade glioma. Br. J. Cancer 119, 693-696 (2018).

65. Fuse MJ, Okada K, Oh-Hara T et al. Mechanisms of resistance to NTRK inhibitors and therapeutic strategies in NTRK1-rearranged cancers. Mol. Cancer Ther. 16, 2130-2143 (2017).

66. Awad MM, Engelman JA, Shaw AT. Acquired resistance to crizotinib from a mutation in CD74-ROS1. N. Engl. J. Med. 369,1173 (2013).

67. Choi YL, Soda M, Yamashita Y et al. EML4-ALK mutations in lung cancer that confer resistance to ALK inhibitors. N. Engl. J. Med. 363, 1734-1739 (2010).

68. Kobayashi S, Boggon TJ, Dayaram T et al. EGFR mutation and resistance of non-small-cell lung cancer to gefitinib. N. Engl. J. Med. 352, 786-792 (2005).

69. Drilon A, Ou SI, Cho BC et al. Repotrectinib (TPX-0005) is a next-generation ROS1/TRK/ALK inhibitor that potently inhibits ROS1/TRK/ALK solvent-front mutations. Cancer Discov. 8, 1227-1236 (2018).

70. Hyman DM, Kummar S, Farago A et al. Phase I and expanded access experience of LOXO-195 (BAY 2731954), a selective next-generation TRK inhibitor (TRKi). Cancer Res. 79(13), Abstract CT127 (2019).

71. Liu D, Offin M, Harnicar S et al. Entrectinib: an orally available, selective tyrosine kinase inhibitor for the treatment of NTRK, ROS1, and ALK fusion-positive solid tumors. Ther. Clin. Risk Manag. 14, 1247-1252 (2018).

72. Lacombe D, Burock S, Bogaerts J et al. The dream and reality of histology agnostic cancer clinical trials. Mol. Oncol. 8, 1057-1063 (2014)

73. Willyard C. 'Basket studies' will hold intricate data for cancer drug approvals. Nat. Med. 19, 655 (2013).

74. Huang FW, Feng FY. A tumor-agnostic NTRK (TRK) inhibitor. Cell 177, 8 (2019).

75. Thomas M, Vora D, Schmidt H. Preparing Health Systems for Tumour-Agnostic Treatment. Report on Readiness of Health Systems for the introduction of Tumour-Agnostic Treatment AT Kearney Germany (2019). https://www.atkearney.de/documents/20152/2291612/Prepari ng+Health+Systems+for+Tumour-Agnostic+Treatment.pdf/90047d16-8d80-b696-1d77-7acc9a5e0e5f?t=1555515831416

76. Hariton E, Locascio JJ. Randomised controlled trials - the gold standard for effectiveness research: study design: randomised controlled trials. BJOG 125,1716 (2018).

77. Offin M, Liu D, Drilon A. Tumor-agnostic drug development. Am. Soc. Clin. Oncol. Educ. Book 38, 184-187 (2018).

78. Marchio C, Scaltriti M, Ladanyi M et al. ESMO recommendations on the standard methods to detect NTRK fusions in daily practice and clinical research. Ann. Oncol. 30, 1417-1427 (2019).

- The European Society of Medical Oncology recommendations on the standard methods to detect NTRK fusions in daily practice and clinical research.

79. National Institute for Health and Care Excellence. Cancer drugs fund. https://www.nice.org.uk/about/what-we-do/our-programmes/nice-guidance/nice-technology-appraisal-guidance/cancer-drugs-fund 